\title{
Stability and Stabilizing of Fractional Complex Lorenz Systems
}

\author{
Rabha W. Ibrahim \\ Institute of Mathematical Sciences, University Malaya, 50603 Kuala Lumpur, Malaysia \\ Correspondence should be addressed to Rabha W. Ibrahim; rabhaibrahim@yahoo.com
}

Received 16 October 2012; Accepted 29 November 2012

Academic Editor: Micah Osilike

Copyright (c) 2013 Rabha W. Ibrahim. This is an open access article distributed under the Creative Commons Attribution License, which permits unrestricted use, distribution, and reproduction in any medium, provided the original work is properly cited.

We study the stability and stabilization of complex fractional Lorenz system. The fractional calculus are taken in sense of the Caputo derivatives. The technique is based on stability theory of fractional-order systems. Numerical solutions are imposed.

\section{Introduction}

Chaos theory is a new branch of mathematics and physics. It provided a new method to understand the behavior of the processes in the world. Chaotic behaviors virtue in different areas of science and engineering such as mechanics, physics, electronics, medicine, biology, ecology, economy. In 1963, Lorenz discovered the chaotic system [1]. A dynamical system is called chaotic if it is deterministic, has long-term aperiodic behavior, and exhibits sensitive dependence on the initial conditions. If the system has one positive Lyapunov exponent, then it is called chaotic. The Lorenz system is a system of ordinary differential equations that imposing chaotic solutions for certain parameter values and initial conditions. Researches along this line ultimately led to the ruling of different classes of relevant chaotic systems. The Lorenz system has advantages, it presents a symmetry, and the equations are invariant under $(x, y) \rightarrow(-x,-y)$. Also the system is dissipative with a value of the divergence and therefore the volume in phase space always contracts under the flow. Moreover the Lorenz system is bounded.

This system was widely considered over the course of the 1970s, 1980s, and 1990s (see [2-5]) as a paradigmatic chaotic problem. In 1994, Sprott defined the diffusionless Lorenz system (a one-parameter version of Lorenz system) [6]. In 1999, Chen and Ueta established an interesting system [7], which is the dual system to the Lorenz system [1], in the sense defined by Vaněček and Čelikovský $[8,9]$. Later, Lü and Chen [10] furthermore introduced a chaotic system, which refers to the transition between the Lorenz and the Chen systems. In 2005, Čelikovský and Chen described another generalized Lorenz canonical form [11]. More systems are posed in 20062007; a unified Lorenz-type system is recognized by Yang et al. [12, 13]. In 2009, Huang and Yang found a new chaotic system, which contains special cases as the modified Lorenz system and conjugate Chen system [14].

Recently, the Lorenz system has got many studies and attentions from researchers. Zhang et al. considered the synchronization of two identical hyperchaotic Lorenz systems with time delay only by a single linear controller [15], while Shi and Wang investigated a class of new synchronization (hybrid synchronization) coexisting in the same system [16]. Camargo et al. considered a system of two identical linearly coupled Lorenz oscillators presenting synchronization of chaotic motion for a specified range of the coupling strength. They verified the existence of global synchronization and antisynchronization attractors with intermingled basins of attraction, such that the basin of one attractor is riddled with holes belonging to the basin of the other attractor and vice versa [17]. Finally, Li et al. concerned with model-free control of the Lorenz chaotic system, where only the online input and output are available, while the mathematic model of the system is unknown [18].

Fractional-order chaotic systems have been studied widely in current years in many fields such as chaotic phenomena, chaotic control, chaotic synchronization, and other related studies. It has been provided that many fractional-order dynamical systems, as some well-known 
integer-order systems, can be displayed complex bifurcation and chaotic phenomena. For example, the fractionalorder Lorenz system, the fractional-order Chen system, the fractional-order Lü system, and the fractional-order unified system also exhibit chaotic behavior.

Čelikovský and Chen (2002) generalized a new Lorenz canonical form of one parameter $[19,20]$. The dynamics of the fractional-order Lorenz model was also investigated by I. Grigorenko and E. Grigorenko (2003) [21]. Li and Peng (2004) studied the time-domain algorithm to compute the chaotic attractor of the fractional differential system, which is completely different from the frequency-domain one [22]. Yan and Li (2007) [23] considered chaos synchronization of fractional-order Lorenz, Rossler, and Chen systems taking one as master and second one as slave. Zhou and Cheng (2008) [24] discussed a synchronization between different fractional-order chaotic systems, namely, Rossler and Chen systems and Chua and Chen systems. Based on the qualitative theory, the existence and uniqueness of solutions for a class of fractional-order Lorenz chaotic systems have been investigated theoretically by Yu et al. (2009) [25]. Fractionalorder diffusion less Lorenz system has been investigated numerically by Sun and Sprott (2009) [26], and results have shown that the system has complex dynamics with interesting characteristics. Recently, Agrawal et al., synchronized fractional-order chaotic systems using active control method [27]. Xu et al., investigated the phenomenon of diffusionless Lorenz system with fractional-order [28]. Zhou and Ding studied the stability and stabilizations of equilibrium points of the fractional-order Lorenz chaotic system [29]. Finally, Si et al. employed an uncertain complex network with four fractional-order Lorenz systems to verify the effectiveness of the proposed approach [30].

In current years, researchers introduced and studied several types of chaotic nonlinear systems with complex variables. These systems which involving complex variables are employed to describe the physics of a detuned laser, rotating fluids, disk dynamos, electronic circuits, and particle beam dynamics in high-energy accelerators. As special model, the chaotic complex Lorenz system which is used to describe and simulate the physics of detuned lasers and thermal convection of liquid flows. The complex Lorenz model is, in fact, the outcome of the study of Baroclinic models. This model corresponds to the equilibrium state of the atmosphere in which surfaces of constant density are not parallel to the surface of constant gravitational potential. In 1982, Fowler et al. defined and studied a complex Lorenz as a generalization of the real Lorenz system $[31,32]$. The complex variables of Lorenz system are related, respectively, to electric field, the atomic polarization amplitudes, and the population inversion in a ring laser system of two-level atoms; for more details, see [33, 34]. Later Rauh et al. (1996) [35] introduced a set of equations to model such a system. This has the same structure as that of Lorenz equations, but it occurs in the complex domain. Panchev and Vitanov (2005) [36] studied asymptotic properties of some complex Lorenz-like systems, while other properties and chaotic synchronization of the complex Lorenz model are studied by Mahmoud et al. (2007)
[37]. Further studies are introduced due to Mahmoud et al.; they constructed the new hyperchaotic complex Lorenz systems by extending the ideas of adding state feedback controls and introducing the complex periodic forcing [38, 39], while G. M. Mahmoud and E. E. Mahmoud (2010) [40] investigated complete synchronization of $n$-dimensional chaotic complex systems with uncertain parameters. Li et al. (2012) [41] considered the distributed robust control problems of uncertain linear multiagent systems with undirected communication topologies. It was assumed that the agents have identical nominal dynamics while subject to different norm-bounded parameter uncertainties, leading to weakly heterogeneous multiagent systems. Mahmoud (2012) [42] introduced a new hyperchaotic complex Lorenz system. This hyperchaotic complex system was constructed by adding a linear controller to the second equation of the chaotic complex Lorenz system. Finally, El-Sayed et al. [43] studied the dynamic properties of the continuous dynamical system of the fractional equations of complex variables. The fivedimensional complex Lorenz model is often used to explain and simulate the physics of tuned lasers. These studies have their origin in the shaping expression by Haken [44] of the isomorphism between the three equations of the real Lorenz model and the three real equations for a single-mode laser operating with its resonant cavity tuned to resonance with the material transition. Furthermore, analytic studies of model systems can be found in [45-47].

In this paper, we study the stability and stabilization of fractional complex Lorenz model. The fractional calculus are taken in sense of the Caputo derivatives. Caputo initial value problem holds for both homogeneous and nonhomogeneous conditions. For this reason choice Caputo derivative is better than other fractional derivatives. The technique based on stability theory of fractional-order systems. Numerical solutions are imposed.

\section{Fractional Calculus}

The idea of the fractional calculus (i.e., calculus of integrals and derivatives of any arbitrary real or complex order) was planted over 300 years ago. Abel in 1823 investigated the generalized tautochrone problem and for the first time applied fractional calculus techniques in a physical problem. Later Liouville applied fractional calculus to problems in potential theory. Since that time the fractional calculus has haggard the attention of many researchers in all area of sciences. Recently, the theory of fractional calculus has found interesting applications in the theory of analytic functions. The classical definitions of fractional operators and their generalizations have fruitfully been applied in obtaining, for example, the characterization properties, coefficient estimates, distortion inequalities, and convolution structures for various subclasses of analytic functions and the works in the research monographs [48-50]. This section concerns with some preliminaries and notations regarding the fractional calculus. Let us start with the Riemann-Liouville fractional operators. 
Definition 1. The fractional (arbitrary) order integral of the function $f$ of order $\alpha>0$ is defined by

$$
I_{a}^{\alpha} f(t)=\int_{a}^{t} \frac{(t-\tau)^{\alpha-1}}{\Gamma(\alpha)} f(\tau) d \tau .
$$

When $a=0$, we write $I_{a}^{\alpha} f(t)=f(t) * \phi_{\alpha}(t)$, where $(*)$ denoted the convolution product, $\phi_{\alpha}(t)=t^{\alpha-1} / \Gamma(\alpha), t>0$, and $\phi_{\alpha}(t)=0, t \leq 0$ and $\phi_{\alpha} \rightarrow \delta(t)$, as $\alpha \rightarrow 0$ where $\delta(t)$ is the delta function.

Definition 2. The fractional (arbitrary) order derivative of the function $f$ of order $0 \leq \alpha<1$ is defined by

$$
D_{a}^{\alpha} f(t)=\frac{d}{d t} \int_{a}^{t} \frac{(t-\tau)^{-\alpha}}{\Gamma(1-\alpha)} f(\tau) d \tau=\frac{d}{d t} I_{a}^{1-\alpha} f(t) .
$$

Remark 3. From Definitions 1 and 2, $a=0$, we have

$$
\begin{gathered}
D^{\alpha} t^{\mu}=\frac{\Gamma(\mu+1)}{\Gamma(\mu-\alpha+1)} t^{\mu-\alpha}, \quad \mu>-1 ; 0<\alpha<1, \\
I^{\alpha} t^{\mu}=\frac{\Gamma(\mu+1)}{\Gamma(\mu+\alpha+1)} t^{\mu+\alpha}, \quad \mu>-1 ; \alpha>0 .
\end{gathered}
$$

Definition 4. The Caputo fractional derivative of order $\mu>0$ is defined, for a smooth function $f(t)$ by

$$
{ }^{c} D^{\mu} f(t):=\frac{1}{\Gamma(n-\mu)} \int_{0}^{t} \frac{f^{(n)}(\zeta)}{(t-\zeta)^{\mu-n+1}} d \zeta
$$

where $n=[\mu]+1$, (the notation $[\mu]$ stands for the largest integer not greater than $\mu$ ).

Remark 5. The following relations hold the following:

(i) Representation

$$
{ }^{c} D^{\mu} f(t)=I^{n-\mu} D^{n} f(t), \quad n-1<\mu<n .
$$

(ii) The Caputo fractional derivative of the power function

$$
{ }^{c} D^{\mu} t^{\mu}=\frac{\Gamma(\mu+1)}{\Gamma(\mu-\alpha+1)} t^{\mu-\alpha}=D^{\alpha} t^{\mu} .
$$

(iii)

$$
I^{\mu c} D^{\mu} f(t)=f(t), \quad f(0)=0, \mu \in(0,1) .
$$

(iv) Linearity

$$
{ }^{c} D^{\mu}(\lambda f(z)+g(z))=\lambda^{c} D_{z}^{\mu} f(t)+{ }^{c} D^{\mu} g(t) .
$$

(v) Noncommutation

$$
{ }^{c} D^{\mu} D^{\alpha} f(t) \neq D^{\alpha}{ }^{c} D^{\mu} f(t)
$$

\section{Stability of Fractional Complex System}

A complex Lorenz-like system has been found in Laser Physics and while analyzing baroclinic instability of the geophysical flows in the atmosphere which takes the form

$$
\begin{gathered}
\xi_{1}=-\sigma \xi_{1}+\sigma \xi_{2}, \\
\xi_{2}=\rho \xi_{1}-a \xi_{2}-\xi_{1} \xi_{3}, \\
\xi_{3}=-b \xi_{3}+\frac{1}{2}\left(\xi_{1}^{*} \xi_{2}+\xi_{1} \xi_{2}^{*}\right),
\end{gathered}
$$

where $\xi_{1}$ and $\xi_{2}$ are complex variables; that is, $\xi_{1}=u_{1}+$ $i u_{2}, \xi_{1}^{*}=u_{1}-i u_{2}$ and $\xi_{2}=u_{3}+i u_{4}, \xi_{2}^{*}=u_{3}-i u_{4}$, but $\xi_{3}:=u_{5}$ is real variable, and $a=1-i \ell$, such that $\rho, \ell, \sigma$, and $b$ are real parameters. It assumed that $u_{k}, k=1, \ldots, 5$ is a function in $t$.

Here, we consider the following fractional complex Lorenz system, in sense of the Caputo derivative:

$$
\begin{gathered}
{ }^{c} D^{\mu_{1}} \xi_{1}=-\sigma \xi_{1}+\sigma \xi_{2}, \\
{ }^{c} D^{\mu_{2}} \xi_{2}=\rho \xi_{1}-a \xi_{2}-\xi_{1} \xi_{3}, \\
{ }^{c} D^{\mu_{3}} \xi_{3}=-b \xi_{3}+\frac{1}{2}\left(\xi_{1}^{*} \xi_{2}+\xi_{1} \xi_{2}^{*}\right) \xi_{3},
\end{gathered}
$$

where $0<\mu_{j} \leq 1, j=1,2,3$; in this note we shall take $\mu_{j} \geq$ 0.95 . For real variables, system (13) reduces to the system of the form

$$
\begin{gathered}
{ }^{c} D^{\mu_{1}} u_{1}=-\sigma u_{1}+\sigma u_{3}, \\
{ }^{c} D^{\mu_{1}} u_{2}=-\sigma u_{2}+\sigma u_{4}, \\
{ }^{c} D^{\mu_{2}} u_{3}=\rho u_{1}-u_{3}-\ell u_{4}-u_{1} u_{5}, \\
{ }^{c} D^{\mu_{2}} u_{4}=\rho u_{2}+\ell u_{3}-u_{4}-u_{2} u_{5}, \\
{ }^{c} D^{\mu_{3}} u_{5}=-b u_{5}+\left(u_{1} u_{3}+u_{2} u_{4}\right) u_{5} .
\end{gathered}
$$

In this section, we shall discuss the stability of the system (14). For this purpose we need the following preliminaries which can be found in [51].

Definition 6. The zero solution of the equation ${ }^{c} D^{\mu} u=$ $f(t, u(t)), \mu \in(0,1]$ is said to be stable if, for any initial values $u_{0}$, there exists $\epsilon>0$ such that $\|u(t)\| \leq \epsilon$, for all $t>t_{0}$. The zero is said to be asymptotically stable if it is stable, and $\|u(t)\| \rightarrow 0, t \rightarrow \infty$.

Lemma 7. Assume the system of the form

$$
{ }^{c} D^{\mu} u=A u(t)+h(u(t)), \quad \mu \in(0,1],
$$

where $u(t)=\left(u_{1}, \ldots, u_{n}(t)\right)^{T} \in \mathbb{R}^{n}, A \in \mathbb{R}^{n \times n}$. If $|\arg (\operatorname{spec}(A))|>\mu \pi / 2, \mu\|A\|>1, \operatorname{spec}(A)$ denotes the eigenvalues of $A$, and $\|\cdot\|$ denotes the $l_{2}$ norm; and $\lim _{u \rightarrow 0}(\|h(u(t))\| /\|u(t)\|)=0$, then the system (15) is asymptotically stable.

Lemma 8. Assume the controller system with the linear feedback control input

$$
{ }^{c} D^{\mu} u=(A+B K) u(t)+h(u(t)), \quad \mu \in(0,1],
$$


where $K \in \mathbb{R}^{1 \times n}$ is a feedback, $B \in \mathbb{R}^{n \times 1}, u(t)=\left(u_{1}, \ldots\right.$, $\left.u_{n}(t)\right)^{T} \in \mathbb{R}^{n}$, and $A \in \mathbb{R}^{n \times n}$. If $|\arg (\operatorname{spec}(A))|>\mu \pi / 2$, $\mu\|A+B K\|>1$, and $\lim _{u \rightarrow 0}(\|h(u(t))\| /\|u(t)\|)=0$, then the system (16) is asymptotically stable.

Theorem 9. If $b>0, \sigma>0$, then the system (14) is asymptotically stable at the equilibrium point $p_{0}=(0,0,0,0,0)$.

Proof. System (14) can be written in the form (15), where

$$
\begin{gathered}
A=\left(\begin{array}{ccccc}
-\sigma & 0 & \sigma & 0 & 0 \\
0 & -\sigma & 0 & \sigma & 0 \\
\rho & 0 & -1 & -\ell & 0 \\
0 & \rho & \ell & -1 & 0 \\
0 & 0 & 0 & 0 & -b
\end{array}\right), \\
h(u)=\left(\begin{array}{c}
0 \\
0 \\
-u_{1} u_{5} \\
-u_{2} u_{5} \\
\left(u_{1} u_{3}+u_{2} u_{4}\right) u_{5}
\end{array}\right) .
\end{gathered}
$$

It is obvious that $h(u)$ satisfies

$$
\begin{aligned}
& \lim _{u \rightarrow 0} \frac{\|h(u(t))\|}{\|u(t)\|} \\
& \quad=\lim _{u \rightarrow 0} \frac{\sqrt{\left(-u_{1} u_{5}\right)^{2}+\left(-u_{2} u_{5}\right)^{2}+\left(u_{5}\right)^{2}\left(u_{1} u_{3}+u_{2} u_{4}\right)^{2}}}{\sqrt{\left(u_{1}\right)^{2}+\left(u_{2}\right)^{2}+\left(u_{3}\right)^{2}+\left(u_{4}\right)^{2}+\left(u_{5}\right)^{2}}} \\
& \leq \lim _{u \rightarrow 0} \frac{\sqrt{\left(-u_{1} u_{5}\right)^{2}+\left(-u_{2} u_{5}\right)^{2}+\left(u_{5}\right)^{2}\left(u_{1} u_{3}+u_{2} u_{4}\right)^{2}}}{\sqrt{u_{5}^{2}}} \\
& \quad=\lim _{u \rightarrow 0} \sqrt{u_{1}^{2}+u_{2}^{2}+\left(u_{1} u_{3}+u_{2} u_{4}\right)^{2}} \\
& =0 .
\end{aligned}
$$

Moreover the characteristic equation of the system is

$$
(\lambda+b)\left[((\sigma+\lambda)(1+\lambda)-\rho \sigma)^{2}+(\sigma+\lambda)^{2} \ell^{2}\right]=0
$$

and when $\ell=0$, the eigenvalues are

$$
\lambda_{1}=-b, \quad \lambda_{2,3}=\frac{-(\sigma+1) \pm \sqrt{(\sigma+1)^{2}-4 \sigma(1-\rho)}}{2}
$$

where $\lambda_{2,3}$ are of multiplicity 2 . Since $-(\sigma+1)<0$, therefore

$$
\left|\arg \left(\lambda_{i}\right)\right|>\frac{\pi}{2}>\frac{\pi}{2} \mu, \quad \mu=\max \left(\mu_{i}, i=1,2,3\right)
$$

and for suitable choice of the parameters $b, \sigma, \ell$, and $\mu_{i}$ we have $\|A\| \mu>1, \mu:=\min \left(\mu_{i}\right)$. According to Lemma 7 , it implies that the equilibrium point $p_{0}$ of system (14) is asymptotically stable. Now for $\ell \neq 0$ and $\rho=0$, the eigenvalues are

$$
\lambda_{1}=-b, \quad \lambda_{2,3}=-\sigma, \quad \lambda_{4,5}=-1 \pm i \ell,
$$

hence relation (16) holds, and consequently the system (14) is asymptotically stable. Finally, the case $\ell \neq 0$ and $\rho \neq 0(\rho>1)$ such that $\sigma=1$, and $\ell^{2}>2(\rho-1)$ implies the eigenvalues of multiplicity 2

$$
\lambda_{1}=-b, \quad \lambda_{2,3}=\frac{-2-\left(\ell^{2}-2 \rho\right) \pm \sqrt{\left(\ell^{2}-2 \rho\right)^{2}-4 \rho^{2}}}{2}
$$

which yields that the system is asymptotically stable at $p_{0}$. Note that the other cases impose complex $\sigma$. This completes the proof.

The system (14) can be assumed in the form (16), we derive the following result.

Theorem 10. If $b>0, \sigma>0$, then the controlled system (14) is asymptotically stable at the equilibrium point $p_{0}=$ $(0,0,0,0,0)$.

Proof. Applying control input $v(t)=B K u(t)$ on (14), where $B=(-\sigma, 0,0,0,0)^{T}$ and $K=(0,0,1,0,0)$ are taken in which $|\arg (\operatorname{spec}(A+B K))|>\mu \pi / 2$, where the characteristic equation of the system is

$$
\begin{aligned}
& (\lambda+b)(\sigma+\lambda)\left[-(\sigma+\lambda)(\lambda+1)^{2}+\sigma \rho(1+\lambda)-\ell^{2}(\sigma+\lambda)\right] \\
& \quad=0 .
\end{aligned}
$$

Obviously, for $\ell=0$ the eigenvalues of the system are

$$
\lambda_{1}=-b, \quad \lambda_{2}=-\sigma, \quad \lambda_{3}=-1,
$$

where $\lambda_{2}$ and $\lambda_{3}$ of multiplicity 2 . Also when $\rho=0$, the eigenvalues are

$$
\lambda_{1}=-b, \quad \lambda_{2}=-\sigma, \quad \lambda_{3}=-1 \pm i \ell .
$$

Finally for $\ell \neq 0, \rho \neq 0$, and $\sigma=1$, the system admits the following eigenvalues:

$$
\lambda_{1}=-b, \quad \lambda_{2,3}=-1, \quad \lambda_{4,5}=-1 \pm \sqrt{\rho-\ell^{2}} .
$$

Furthermore, $\mu\|A+B K\|>1$; hence in view of Lemma 8, controlled system (14) is asymptotically stable at $p_{0}$.

\section{Stabilizing $p_{0}$}

In this section, we design an evaluation controller for fractional-order Lorenz chaotic system (14) via fractionalorder derivative. For this puropose we need the following result which can be found in [52]. 
Lemma 11. The fixed points of the following nonlinear commensurate fractional-order autonomous system,

$$
{ }^{c} D^{\mu} u=f(u), \quad \mu \in(0,1),
$$

are asymptotically stable if all eigenvalues $(\lambda)$ of the Jacobian matrix evaluated at the fixed points satisfy $|\arg \lambda|>0.5 \pi \mu$, where $0<\mu<1, u \in R^{n}, f: R^{n} \rightarrow R^{n}$ are continuous nonlinear vector functions, and the fixed points of this nonlinear commensurate fractional-order system are calculated by solving equation $f(u)=0$.

We can obtain the following results.

Theorem 12. Assume the controlled fractional-order Lorenz chaotic system

$$
\begin{gathered}
{ }^{c} D^{\mu_{1}} u_{1}=-\sigma u_{1}+\sigma u_{3}, \\
{ }^{c} D^{\mu_{1}} u_{2}=-\sigma u_{2}+\sigma u_{4}, \\
{ }^{c} D^{\mu_{2}} u_{3}=\rho u_{1}-u_{3}-\ell u_{4}-u_{1} u_{5}+f_{1}\left(u_{1}\right), \\
{ }^{c} D^{\mu_{2}} u_{4}=\rho u_{2}+\ell u_{3}-u_{4}-u_{2} u_{5}, \\
{ }^{c} D^{\mu_{3}} u_{5}=-b u_{5}+\left(u_{1} u_{3}+u_{2} u_{4}\right) u_{5},
\end{gathered}
$$

where $f_{1}\left(u_{1}\right)=-k_{11}{ }^{c} D^{\mu_{1}} u_{1}-k_{12} u_{1}$ is the fractional-order controller, and $k_{1 i}, i=1,2$ is the feedback coefficient. For the case $\ell=0$, if $b>0, \sigma>0$,

$$
1+k_{11} \sigma>0, \quad k_{12}=\rho+k_{11} \sigma
$$

then system (30) will asymptotically converge to the unstable equilibrium point $p_{0}$. Furthermore, for the case $\ell \neq 0, \rho \neq 0$, and $\sigma=1$, if $b>0$ and $k_{11}>-3$, then system (30) will asymptotically converge to the unstable equilibrium point $p_{0}$.

Proof. The Jacobi matrix of the controlled fractional-order Lorenz chaotic system $(30)$ at $p_{0}$ is

$$
J=\left(\begin{array}{ccccc}
-\sigma & 0 & \sigma & 0 & 0 \\
0 & -\sigma & 0 & \sigma & 0 \\
\rho+k_{11} \sigma-k_{12} & 0 & -1-k_{11} \sigma & -\ell & 0 \\
0 & \rho & \ell & -1 & 0 \\
0 & 0 & 0 & 0 & -b
\end{array}\right) .
$$

Since $k_{12}=\rho+k_{11} \sigma$, we have

$$
J=\left(\begin{array}{ccccc}
-\sigma & 0 & \sigma & 0 & 0 \\
0 & -\sigma & 0 & \sigma & 0 \\
0 & 0 & -1-k_{11} \sigma & -\ell & 0 \\
0 & \rho & \ell & -1 & 0 \\
0 & 0 & 0 & 0 & -b
\end{array}\right)
$$

Thus the characteristic equation takes the form

$$
\begin{aligned}
(b+\lambda)[ & (\sigma+\lambda)^{2}(1+\lambda)\left(1+k_{11} \sigma+\lambda\right) \\
& \left.-(\sigma+\lambda) \rho \sigma\left(1+k_{11} \sigma+\lambda\right)+(\sigma+\lambda)^{2} \ell^{2}\right]=0
\end{aligned}
$$

which leads to the eigenvalues

$$
\begin{aligned}
& \lambda_{1}=-b, \quad \lambda_{2}=-\sigma, \quad \lambda_{3}=-1-k_{11} \sigma \\
& \lambda_{4,5}=\frac{-(\sigma+1) \pm \sqrt{(\sigma+1)^{2}-4 \sigma(1-\rho)}}{2}
\end{aligned}
$$

when $\ell=0$. And for $\rho=0$, we have

$$
\begin{gathered}
\lambda_{1}=-b, \quad \lambda_{2,3}=-\sigma \\
\lambda_{4,5}=\frac{-\left(2+k_{11} \sigma\right) \pm \sqrt{\left(2+k_{11} \sigma\right)^{2}-4\left(\ell^{2}+k_{11} \sigma+1\right)}}{2} .
\end{gathered}
$$

Since $b>0, \sigma>0$, and $1+k_{11} \sigma>0$, therefore,

$$
\left|\arg \lambda_{i}\right|>0.5 \pi \mu, \quad i=1, \ldots, 5 \text {, }
$$

where $\mu:=\max \mu_{j}$, and $j=1,2,3$. According to Lemma 11, it yields that the equilibrium point $p_{0}$ of system (30) is asymptotically stable; that is, the unstable equilibrium point $p_{0}$ in fractional-order Lorenz system (30) can be stabilized via fractional-order derivative. For the case $\ell \neq 0, \rho \neq 0$, and $\sigma=1$, since $k_{11}>-3$, then the characteristic equation

$$
\begin{gathered}
\lambda^{3}+\left(3+k_{11}\right) \lambda^{2}+\left(3+2 k_{11}-\rho+\ell^{2}\right) \lambda \\
+\left(1+k_{11}-\rho-\rho k_{11}+\ell^{2}\right)=0
\end{gathered}
$$

has negative eigenvalues, and hence the system is asymptotically stable. The proof is completed.

Theorem 13. Assume the controlled fractional-order Lorenz chaotic system

$$
\begin{gathered}
{ }^{c} D^{\mu_{1}} u_{1}=-\sigma u_{1}+\sigma u_{3}, \\
{ }^{c} D^{\mu_{1}} u_{2}=-\sigma u_{2}+\sigma u_{4}, \\
{ }^{c} D^{\mu_{2}} u_{3}=\rho u_{1}-u_{3}-\ell u_{4}-u_{1} u_{5}, \\
{ }^{c} D^{\mu_{2}} u_{4}=\rho u_{2}+\ell u_{3}-u_{4}-u_{2} u_{5}+f_{2}\left(u_{2}\right), \\
{ }^{c} D^{\mu_{3}} u_{5}=-b u_{5}+\left(u_{1} u_{3}+u_{2} u_{4}\right) u_{5},
\end{gathered}
$$

where $f_{2}\left(u_{2}\right)=-k_{21}{ }^{c} D^{\mu_{1}} u_{2}-k_{22} u_{2}$ is the fractional-order controller, and $k_{2 i}, i=1,2$ is the feedback coefficient. For the case $\ell=0$, if $b>0, \sigma>0$,

$$
1+k_{21} \sigma>0, \quad k_{22}=\rho+k_{21} \sigma,
$$

then system (39) will asymptotically converge to the unstable equilibrium point $p_{0}$. Furthermore, for the case $\ell \neq 0, \rho \neq 0$, and $\sigma=1$, if $b>0$ and $k_{21}>-3$, then system (39) will asymptotically converge to the unstable equilibrium point $p_{0}$.

Proof. The Jacobi matrix of the controlled fractional-order Lorenz chaotic system (39) at $p_{0}$ is

$$
J=\left(\begin{array}{ccccc}
-\sigma & 0 & \sigma & 0 & 0 \\
0 & -\sigma & 0 & \sigma & 0 \\
\rho & 0 & -1 & -\ell & 0 \\
0 & \rho+k_{21} \sigma-k_{22} & \ell & -\left(1+\sigma k_{21}\right) & 0 \\
0 & 0 & 0 & 0 & -b
\end{array}\right) .
$$


Since $k_{22}=\rho+k_{21} \sigma$, we have

$$
J=\left(\begin{array}{ccccc}
-\sigma & 0 & \sigma & 0 & 0 \\
0 & -\sigma & 0 & \sigma & 0 \\
\rho & 0 & -1 & -\ell & 0 \\
0 & 0 & \ell & -\left(1+\sigma k_{21}\right) & 0 \\
0 & 0 & 0 & 0 & -b
\end{array}\right)
$$

Therefore, the characteristic equation is

$$
\begin{aligned}
& (b+\lambda)(\sigma+\lambda)\left[(\sigma+\lambda)(1+\lambda)\left(1+k_{21} \sigma+\lambda\right)\right. \\
& \left.+\rho \sigma\left(1+k_{21} \sigma+\lambda\right)-(\sigma+\lambda) \ell^{2}\right]=0
\end{aligned}
$$

which yields the eigenvalues

$$
\begin{aligned}
& \lambda_{1}=-b, \quad \lambda_{2}=-\sigma, \quad \lambda_{3}=-1-k_{21} \sigma, \\
& \lambda_{4,5}=\frac{-(\sigma+1) \pm \sqrt{(\sigma+1)^{2}-4 \sigma(1+\rho)}}{2}
\end{aligned}
$$

when $\ell=0$. while for $\rho=0$, we impose

$$
\begin{gathered}
\lambda_{1}=-b, \quad \lambda_{2,3}=-\sigma \\
\lambda_{4,5}=\frac{-\left(2+k_{21} \sigma\right) \pm \sqrt{\left(2+k_{21} \sigma\right)^{2}-4\left(1-\ell^{2}+k_{21} \sigma\right)}}{2}
\end{gathered}
$$

Since $b>0, \sigma>0$, and $1+k_{21} \sigma>0$, therefore,

$$
\left|\arg \lambda_{i}\right|>0.5 \pi \mu, \quad i=1, \ldots, 5,
$$

where $\mu:=\max \mu_{j}, j=1,2,3$. According to Lemma 11, it yields that the equilibrium point $p_{0}$ of system (39) is asymptotically stable; that is, the unstable equilibrium point $p_{0}$ in fractional-order Lorenz system (39) can be stabilized via fractional-order derivative. For the case $\ell \neq 0, \rho \neq 0$, and $\sigma=1$, since $k_{21}>-3$, then the characteristic equation

$$
\begin{gathered}
\lambda^{3}+\left(3+k_{21}\right) \lambda^{2}+\left(3+2 k_{21}+\rho-\ell^{2}\right) \lambda \\
+\left(1+k_{21}(1+\rho)-\ell^{2}\right)=0
\end{gathered}
$$

has negative eigenvalues, and hence the system is asymptotically stable. This completes the proof.

Theorem 14. Assume the controlled fractional-order Lorenz chaotic system

$$
\begin{gathered}
{ }^{c} D^{\mu_{1}} u_{1}=-\sigma u_{1}+\sigma u_{3}+f_{3}\left(u_{3}\right), \\
{ }^{c} D^{\mu_{1}} u_{2}=-\sigma u_{2}+\sigma u_{4}, \\
{ }^{c} D^{\mu_{2}} u_{3}=\rho u_{1}-u_{3}-\ell u_{4}-u_{1} u_{5}, \\
{ }^{c} D^{\mu_{2}} u_{4}=\rho u_{2}+\ell u_{3}-u_{4}-u_{2} u_{5}, \\
{ }^{c} D^{\mu_{3}} u_{5}=-b u_{5}+\left(u_{1} u_{3}+u_{2} u_{4}\right) u_{5},
\end{gathered}
$$

where $f_{3}\left(u_{3}\right)=-k_{31}{ }^{c} D^{\mu_{2}} u_{3}-k_{32} u_{3}$ is the fractional-order controller, and $k_{2 i}, i=1,2$ is the feedback coefficient. If $\ell=$ $0, b>0, \sigma>0, k_{32}=\sigma+k_{31}$, and $\sigma+\rho k_{31}>0$, then system (48) will asymptotically converge to the unstable equilibrium point $p_{0}$.

Proof. The Jacobi matrix of the controlled fractional-order Lorenz chaotic system (48) at $p_{0}$ is

$$
J=\left(\begin{array}{ccccc}
-\sigma-\rho k_{31} & 0 & \sigma+k_{31}-k_{32} & \ell k_{31} & 0 \\
0 & -\sigma & 0 & \sigma & 0 \\
\rho & 0 & -1 & -\ell & 0 \\
0 & \rho & \ell & -1 & 0 \\
0 & 0 & 0 & 0 & -b
\end{array}\right)
$$

Because $\ell=0, k_{32}=\sigma+k_{31} \sigma$, so we have

$$
J=\left(\begin{array}{ccccc}
-\sigma-\rho k_{31} & 0 & 0 & 0 & 0 \\
0 & -\sigma & 0 & \sigma & 0 \\
\rho & 0 & -1 & 0 & 0 \\
0 & \rho & 0 & -1 & 0 \\
0 & 0 & 0 & 0 & -b
\end{array}\right)
$$

The characteristic equation is

$$
(b+\lambda)\left(\sigma+\rho k_{31}+\lambda\right)(1+\lambda)[(\sigma+\lambda)(1+\lambda)-\rho \sigma]=0
$$

which implies the eigenvalues

$$
\begin{gathered}
\lambda_{1}=-b, \quad \lambda_{2}=-\sigma, \quad \lambda_{3}=-1, \\
\lambda_{4,5}=\frac{-(\sigma+1) \pm \sqrt{(\sigma+1)^{2}-4 \sigma(1-\rho)}}{2} .
\end{gathered}
$$

By the assumptions of the theorem and in virtue of Lemma 11, we obtain that system (48) will asymptotically converge to the unstable equilibrium point $p_{0}$.

In the same manner of Theorem 14, we have the following result.

Theorem 15. Assume the controlled fractional-order Lorenz chaotic system

$$
\begin{gathered}
{ }^{c} D^{\mu_{1}} u_{1}=-\sigma u_{1}+\sigma u_{3}, \\
{ }^{c} D^{\mu_{1}} u_{2}=-\sigma u_{2}+\sigma u_{4}+f_{4}\left(u_{4}\right), \\
{ }^{c} D^{\mu_{2}} u_{3}=\rho u_{1}-u_{3}-\ell u_{4}-u_{1} u_{5}, \\
{ }^{c} D^{\mu_{2}} u_{4}=\rho u_{2}+\ell u_{3}-u_{4}-u_{2} u_{5}, \\
{ }^{c} D^{\mu_{3}} u_{5}=-b u_{5}+\left(u_{1} u_{3}+u_{2} u_{4}\right) u_{5},
\end{gathered}
$$


where $f_{4}\left(u_{4}\right)=-k_{41}{ }^{c} D^{\mu_{2}} u_{4}-k_{42} u_{4}$ is the fractional-order controller, and $k_{2 i}, i=1,2$ is the feedback coefficient. If $\ell=$ $0, b>0, \sigma>0, k_{42}=\sigma+k_{41}$, and $\sigma+\rho k_{41}>0$, then system (53) will asymptotically converge to the unstable equilibrium point $p_{0}$.

\section{Numerical Solution}

In this section, we discuss the numerical solution of fractional differential equations. All the numerical simulations of fractional-order system in this paper are based on [22]. Set $h=T / N, t_{n}=n h$, and $n=1, \ldots, N$ with the initial condition $\left(u_{1}(0), \ldots, u_{5}(0)\right)$, therefore, the system (14) can be described as follows:

$u_{1}(n+1)$

$$
\begin{aligned}
=u_{1}(0)+\frac{h^{\mu_{1}}}{\Gamma\left(\mu_{1}+2\right)}\left[\sigma\left(-u_{1}^{p}(n+1)+u_{3}^{p}(n+1)\right),\right. \\
\left.+\sum_{j=0}^{n} A_{1, j, n+1} \times \sigma\left(-u_{1}(j)+u_{3}(j)\right)\right]
\end{aligned}
$$

$u_{2}(n+1)$

$$
\begin{aligned}
=u_{2}(0)+\frac{h^{\mu_{1}}}{\Gamma\left(\mu_{1}+2\right)}\left[\sigma\left(-u_{2}^{p}(n+1)+u_{4}^{p}(n+1)\right),\right. \\
\left.+\sum_{j=0}^{n} A_{2, j, n+1} \times \sigma\left(-u_{2}(j)+u_{4}(j)\right)\right]
\end{aligned}
$$

$u_{3}(n+1)$

$$
\begin{aligned}
= & u_{3}(0)+\frac{h^{\mu_{2}}}{\Gamma\left(\mu_{2}+2\right)} \\
\times & {\left[\left(\rho u_{1}^{p}(n+1)-u_{3}^{p}(n+1)\right.\right.} \\
& \left.-\ell u_{4}^{p}(n+1)-u_{1}^{p}(n+1) u_{5}^{p}(n+1)\right) \\
& \left.+\sum_{j=0}^{n} A_{3, j, n+1}\left(\rho u_{1}(j)-u_{3}(j)-\ell u_{4}(j)-u_{1}(j) u_{5}(j)\right)\right],
\end{aligned}
$$$$
u_{4}(n+1)
$$$$
=u_{4}(0)+\frac{h^{\mu_{2}}}{\Gamma\left(\mu_{2}+2\right)}
$$$$
\times\left[\left(\rho u_{2}^{p}(n+1)-u_{4}^{p}(n+1)+\ell u_{3}^{p}(n+1)\right.\right.
$$

$$
\begin{aligned}
& \left.-u_{2}^{p}(n+1) u_{5}^{p}(n+1)\right) \\
& \left.+\sum_{j=0}^{n} A_{4, j, n+1}\left(\rho u_{2}(j)-u_{4}(j)+\ell u_{3}(j)-u_{2}(j) u_{5}(j)\right)\right], \\
& u_{5}(n+1) \\
& =u_{5}(0)+\frac{h^{\mu_{3}}}{\Gamma\left(\mu_{3}+2\right)} \\
& \times\left[\left(-b u_{5}^{p}(n+1)\right.\right. \\
& +\left(u_{1}^{p}(n+1) u_{3}^{p}(n+1)+u_{2}^{p}(n+1) u_{4}^{p}(n+1)\right) \\
& \left.\times u_{5}^{p}(n+1)\right)+\sum_{j=0}^{n} A_{5, j, n+1} \\
& \left.\times\left(-b u_{5}(j)+\left(u_{1}(j) u_{3}(j)+u_{2}(j) u_{4}(j)\right) u_{5}(j)\right)\right], \\
& u_{1}^{p}(n+1)=u_{1}(0)+\sum_{j=0}^{n} B_{1, j, n+1} \times \sigma\left(-u_{1}(j)+u_{3}(j)\right) \text {, } \\
& u_{2}^{p}(n+1)=u_{2}(0)+\sum_{j=0}^{n} B_{2, j, n+1} \times \sigma\left(-u_{2}(j)+u_{4}(j)\right) \text {, } \\
& u_{3}^{p}(n+1)=u_{3}(0)+\sum_{j=0}^{n} B_{3, j, n+1} \\
& \times\left(\rho u_{1}(j)-u_{3}(j)-\ell u_{4}(j)-u_{1}(j) u_{5}(j)\right), \\
& u_{4}^{p}(n+1)=u_{4}(0)+\sum_{j=0}^{n} B_{4, j, n+1} \\
& \times\left(\rho u_{2}(j)-u_{4}(j)+\ell u_{3}(j)-u_{2}(j) u_{5}(j)\right), \\
& u_{5}^{p}(n+1) \\
& =u_{5}(0)+\sum_{j=0}^{n} B_{5, j, n+1} \\
& \times\left(-b u_{5}(j)+\left(u_{1}(j) u_{3}(j)+u_{2}(j) u_{4}(j)\right) u_{5}(j)\right),
\end{aligned}
$$

and for $k=1, \ldots, 5$

$A_{k, j, n+1}$

$$
\begin{aligned}
& = \begin{cases}n^{\mu+1}-(n-\mu)(n+1)^{\mu}, & j=0, \\
(n-j+2)^{\mu+1}+(n-j)^{\mu+1} & \\
-2(n-j+2)^{\mu+1}, & 1 \leq j \leq n, \\
1, & j=n+1,\end{cases} \\
& B_{k, j, n+1}=\frac{h^{\mu}}{\mu}\left[(n-j+1)^{\mu}-(n-j)^{\mu}\right], \quad 0 \leq j \leq n \text {. }
\end{aligned}
$$




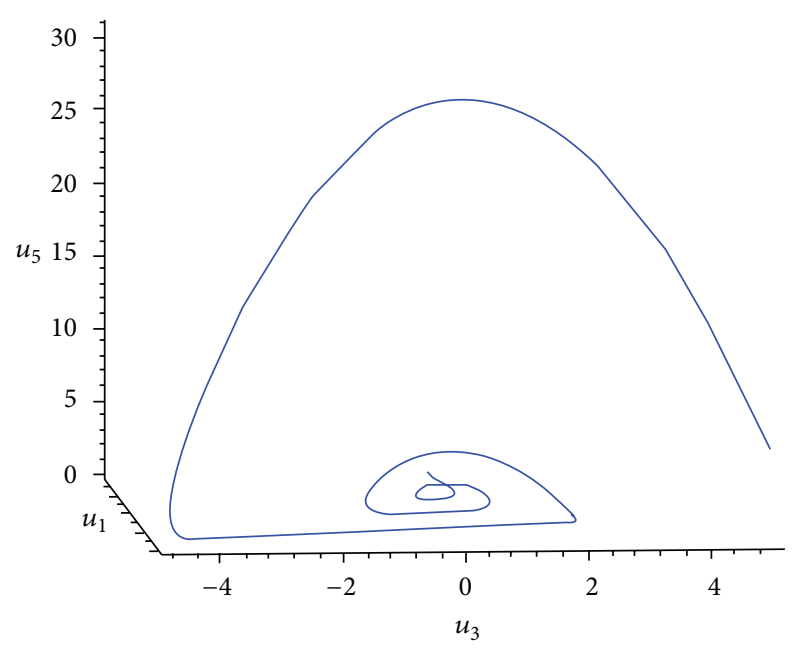

(a)

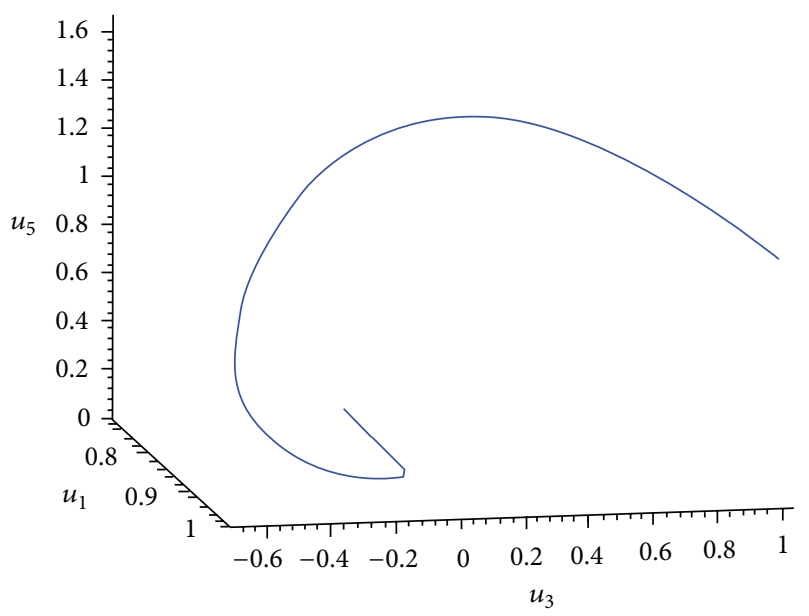

(c)

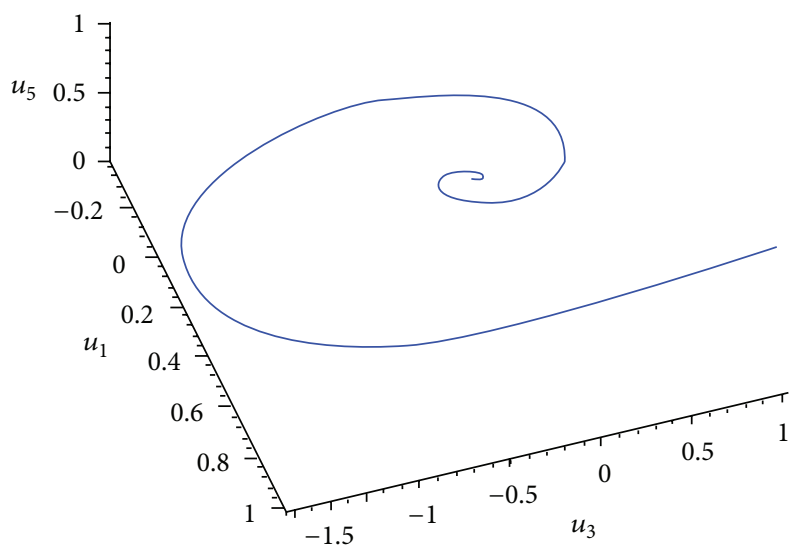

(b)

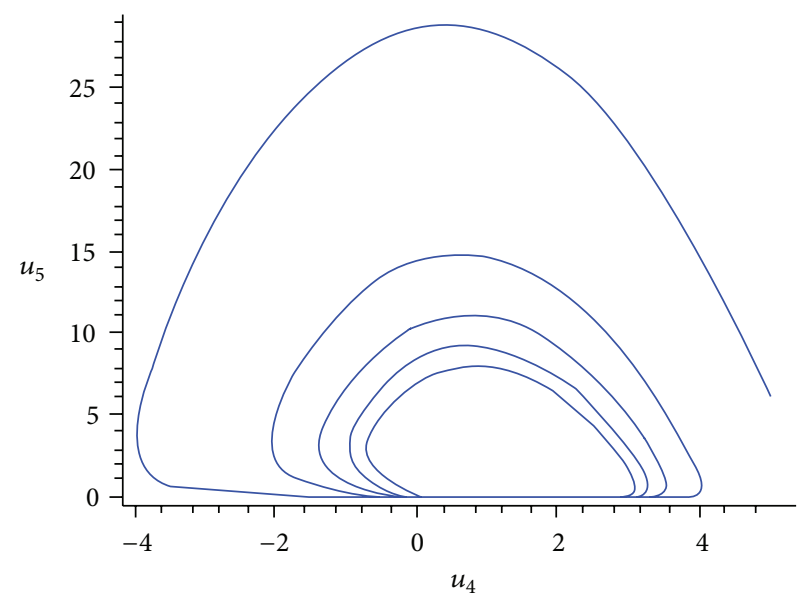

(d)

Figure 1: Asymptotically stable at $p_{0}$.

The error of this approximation can be computed as follows:

$$
\left|u_{i}\left(t_{n}\right)-u_{i}(n)\right|=o\left(h^{\mu}\right), \quad p=\min \left(2,1+\max \mu_{1,2,3}\right) .
$$

\section{Synchronizing $p_{0}$}

we consider a feedback controller for the fractional-order Lorenz chaotic system (14) via fractional-order derivative and obtain the controlled response system

$$
\begin{gathered}
{ }^{c} D^{\mu_{1}} v_{1}=-\sigma v_{1}+\sigma v_{3}, \\
{ }^{c} D^{\mu_{1}} v_{2}=-\sigma v_{2}+\sigma v_{4}, \\
{ }^{c} D^{\mu_{2}} v_{3}=\rho v_{1}-v_{3}-\ell v_{4}-v_{1} v_{5}+V, \\
{ }^{c} D^{\mu_{2}} v_{4}=\rho v_{2}+\ell v_{3}-v_{4}-v_{2} v_{5}, \\
{ }^{c} D^{\mu_{3}} v_{5}=-b v_{5}+\left(v_{1} v_{3}+v_{2} v_{4}\right) v_{5},
\end{gathered}
$$

where

$$
\begin{aligned}
V= & k_{1}\left[{ }^{c} D^{\mu_{1}} v_{1}-D^{\mu_{1}} u_{1}\right]+k_{2}\left(v_{1}-u_{1}\right) \\
& +v_{1} v_{5}-\rho u_{1}+u_{3}+\ell u_{4}
\end{aligned}
$$

is the fractional-order controller, and $k_{i}, i=1,2$ is the feedback coefficient. We need the following result which can be found in [53].

Lemma 16. The following linear commensurate fractionalorder autonomous system,

$$
{ }^{c} D^{\mu} u=A u, \quad \mu \in(0,1),
$$

is asymptotically stable if and only if $|\arg \lambda|>0.5 \pi \mu$ is satisfied for all eigenvalues $(\lambda)$ of matrix $A$. Also, this system is stable if and only if $|\arg \lambda| \geq 0.5 \pi \mu$ is satisfied for all eigenvalues $(\lambda)$ of matrix $A$, and those critical eigenvalues which satisfy $|\arg \lambda|=$ 


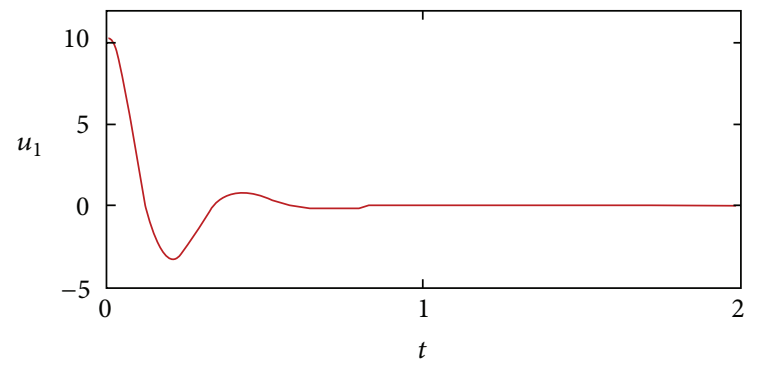

(a)

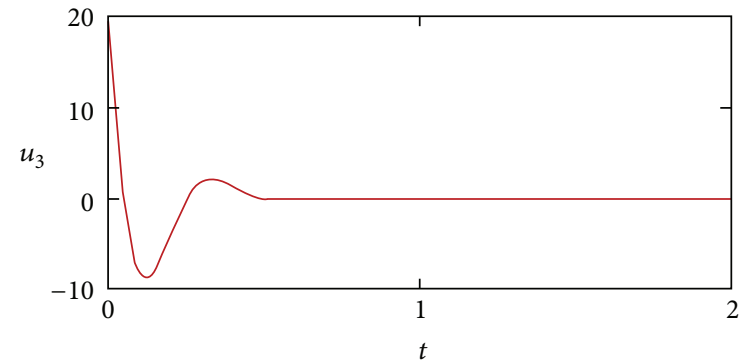

(b)

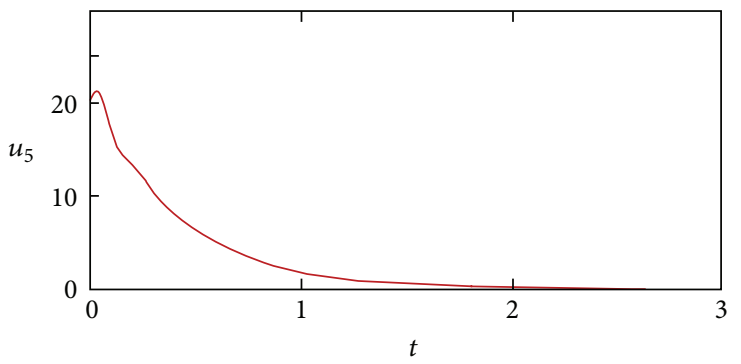

(c)

Figure 2: Time evolution for $\left(u_{1}, u_{3}\right.$, and $\left.u_{5}\right)$ at $p_{0}$.

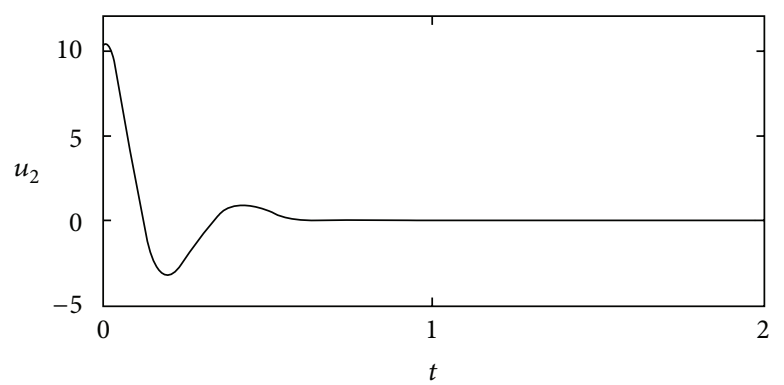

(a)

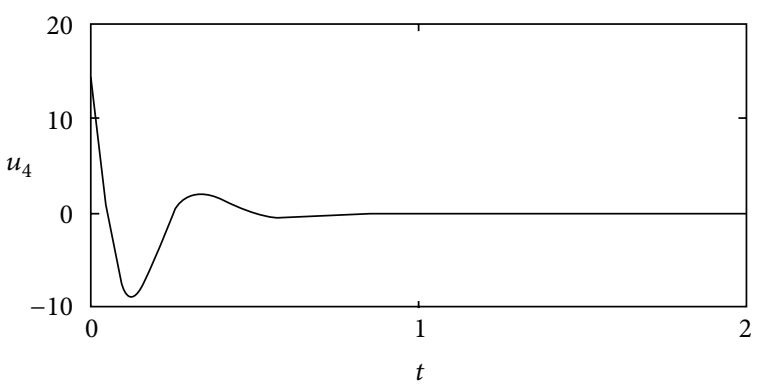

(b)

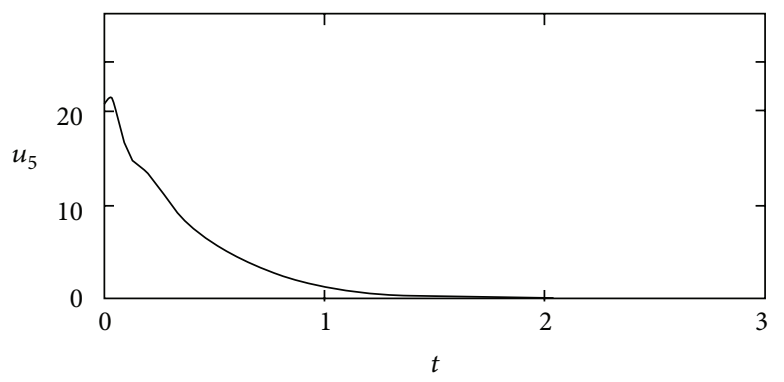

(c)

FIgURE 3: Time evolution for $\left(u_{2}, u_{4}\right.$, and $\left.u_{5}\right)$ at $p_{0}$. 


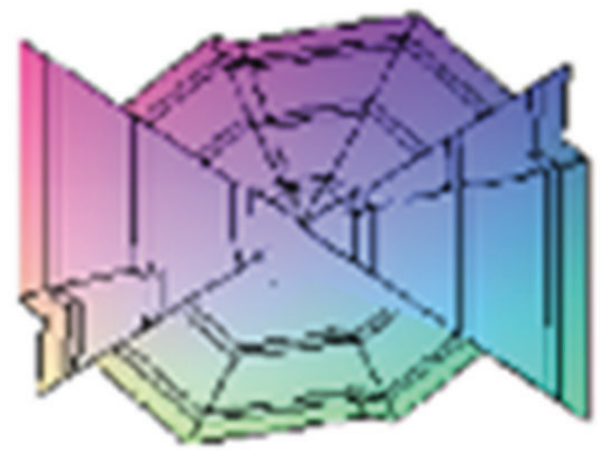

Figure 4: $(10,28,8 / 3,0)$.

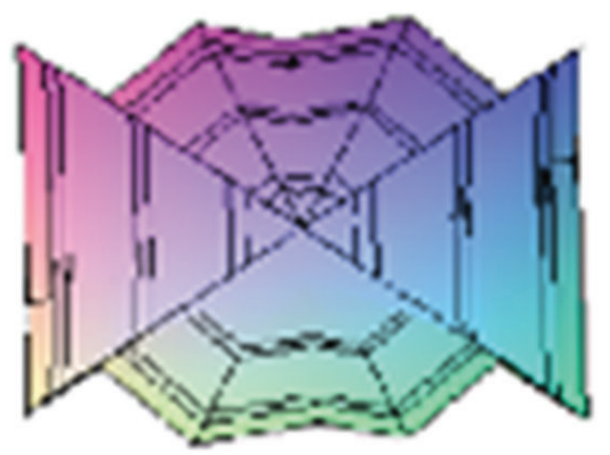

Figure 5: $(10,28,8 / 3,1)$.

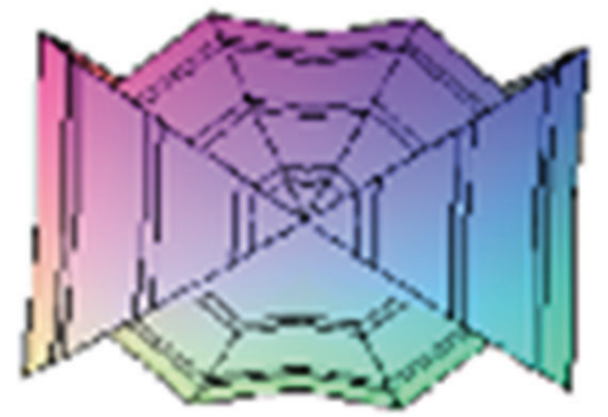

Figure 6: $(10,28,8 / 3,10)$.

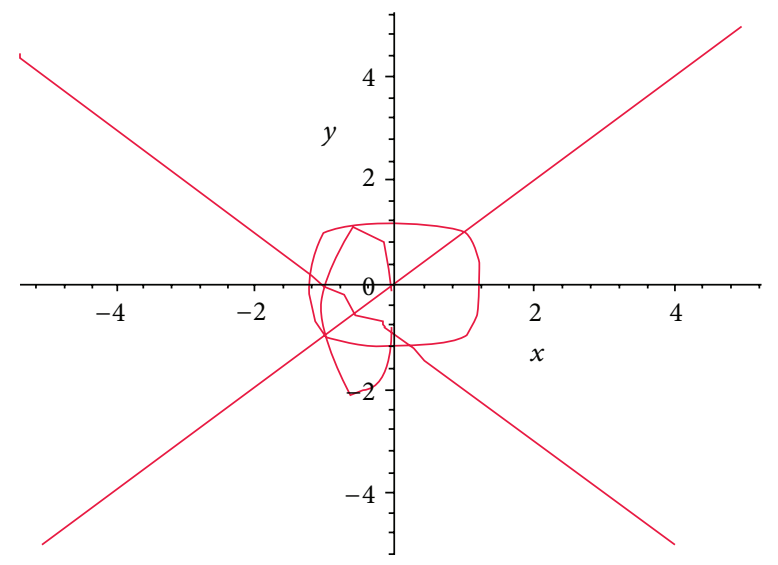

Figure 7: The phase plane of $p_{0}$. 


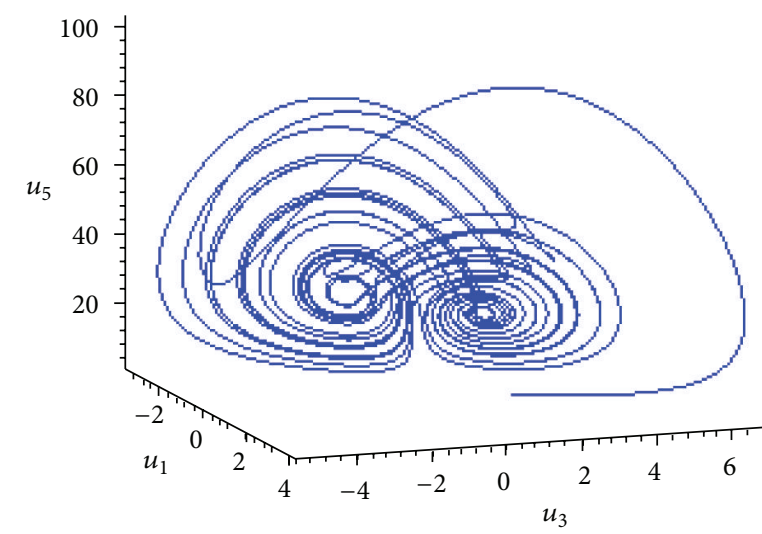

(a)

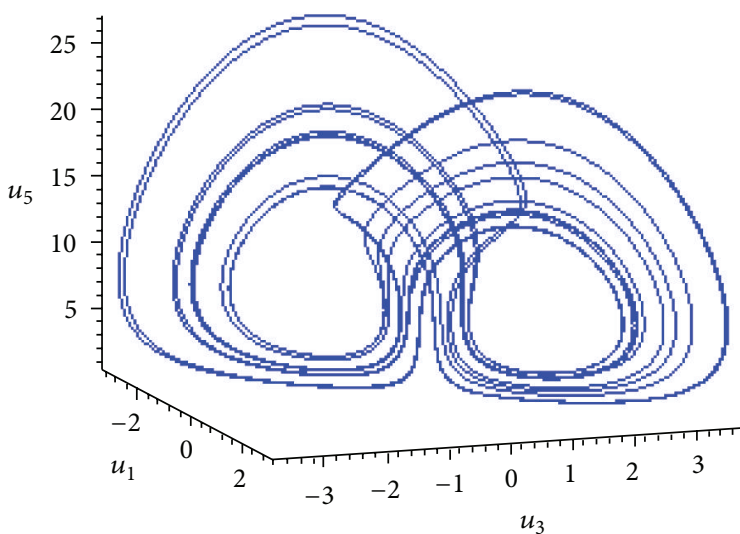

(c)

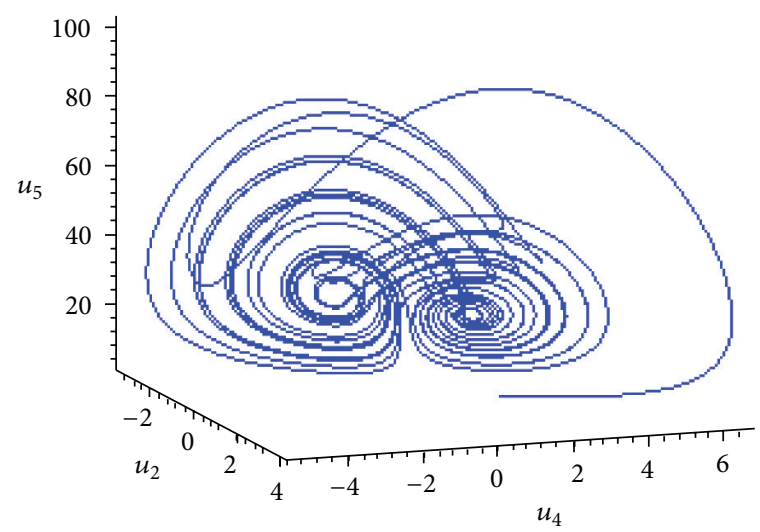

(b)

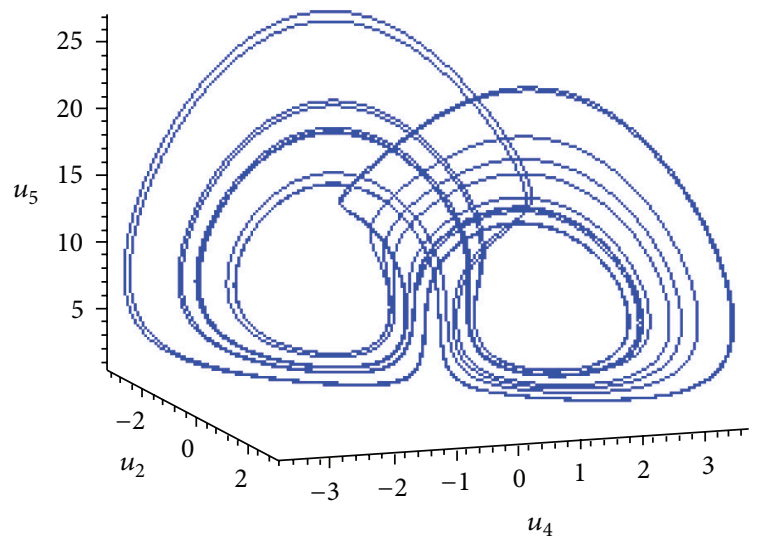

(d)

FIGURE 8: Chaotic attractors of the proposed system.

$0.5 \pi \mu$ have geometric multiplicity one, where $0<\mu<1, u \in$ $R^{n}$, and $A \in R^{n} \times R^{n}$.

We have the following theorem.

Theorem 17. If $b>0, \sigma>0, k_{2}=-\rho+k_{1} \sigma$, and $\sigma\left(k_{1}+\rho\right)<1$, then the fractional-order Lorenz chaotic system (14) and the controlled fractional-order Lorenz chaotic system (58) achieved synchronization via fractional-order derivative.

Proof. Define the synchronization error variables as follows:

$$
e_{j}=v_{j}-u_{j}, \quad j=1, \ldots, 5 \text {. }
$$

Therefore, we obtain the system

$$
\left(\begin{array}{l}
{ }^{c} D^{\mu_{1}} e_{1} \\
{ }^{c} D^{\mu_{1}} e_{2} \\
{ }^{c} D^{\mu_{2}} e_{3} \\
{ }^{c} D^{\mu_{2}} e_{4} \\
{ }^{c} D^{\mu_{3}} e_{5}
\end{array}\right)=A\left(\begin{array}{l}
e_{1} \\
e_{2} \\
e_{3} \\
e_{4} \\
e_{5}
\end{array}\right)
$$

where

A

$$
=\left(\begin{array}{ccccc}
-\sigma & 0 & \sigma & 0 & 0 \\
0 & -\sigma & 0 & \sigma & 0 \\
\rho-k_{1} \sigma+k_{2} & 0 & -1+k_{1} \sigma & -\ell & 0 \\
0 & \rho-u_{5} & \ell & -1 & -v_{2} \\
u_{5} u_{3} & u_{5} u_{4} & u_{5} v_{1} & u_{5} v_{2} & -b+v_{1} v_{3}+v_{2} v_{4}
\end{array}\right) .
$$

Consider $u_{i}$ and $v_{i}$ with $k_{2}=-\rho+k_{1} \sigma$, such that

$$
A=\left(\begin{array}{ccccc}
-\sigma & 0 & \sigma & 0 & 0 \\
0 & -\sigma & 0 & \sigma & 0 \\
0 & 0 & -1+k_{1} \sigma & -\ell & 0 \\
0 & \rho & \ell & -1 & 0 \\
0 & 0 & 0 & 0 & -b
\end{array}\right)
$$


Thus, the characteristic equation is of the form

$$
\begin{aligned}
& (b+\lambda)(\sigma+\lambda)\left[(\sigma+\lambda)(1+\lambda)\left(k_{1} \sigma-\lambda-1\right)\right. \\
& \left.-\rho \sigma(1+\lambda)\left(k_{1} \sigma-\lambda-1\right)-\ell^{2}(\sigma+\lambda)\right]=0 .
\end{aligned}
$$

Because $\sigma\left(k_{1}+\rho\right)<1$ yields that $\left|\arg \lambda_{i}(A)\right|>\pi / 2>$ $(\pi / 2) \mu, i=1, \ldots, 5$. According to Lemma 16, it implies that the equilibrium point $p_{0}$ of error system (60) is asymptotically stable,and hence the fractional-order Lorenz chaotic system (14) and the controlled fractional-order Lorenz chaotic system (58) achieved synchronization via fractionalorder derivative. The proof is completed.

\section{Conclusion and Discussion}

The five-dimensional complex models are habitually used to clarify and simulate the physics of tuned lasers. By using fractional-order derivative (in sense of the Caputo derivatives), we stabilized the unstable equilibrium points of the complex fractional-order Lorenz chaotic system and included chaos synchronization for the fractional-order Lorenz chaotic system. Numerical solutions are computed for these systems. Conditions are imposed to study the stability, and several cases are discussed for these systems. Figure 1 shows the asymptotically stable at $p_{0}$, such that $\sigma>0, b>$ 0 , and the values of $\rho$ are taken positive in (a) with the initial values $(4,5,6)$, negative in (b) with the initial values $(1,1,1)$, and for $\rho=0$ in (c), while in (d) the parameters are valued as $\sigma=1, \rho=2$, and $b=3$ with the initial values $(4,5,6)$. Figures 2 and 3 confirm the time evolution at $p_{0}$. Figures $4-6$ show the affectedness of $\ell=0,1,10$, where $(\sigma, \rho, b, \ell)=(10,28,8 / 3,0)$ in Figure $4,(10,28,8 / 3,1)$ in Figure 5 , and $(10,28,8 / 3,10)$ in Figure 6 . Figure 7 shows the phase plane of $p_{0}$. Here we discuss the system for $\mu_{i}=$ 0.998, $i=1,2,3$. Note that $u_{2}$ and $u_{4}$ are congruent on $u_{1}$ and $u_{3}$, respectively. Finally, Figure 8 represents to the chaotic attractors of the proposed system with the parameters $(\sigma, \rho, b, \ell)=(10,28,8 / 3,0)$ and $(\sigma, \rho, b, \ell)=(10,8,8 / 3,0)$ with the initial values $(1,2,3)$, respectively.

\section{References}

[1] E. N. Lorenz, "Deterministic nonperiodic flow," Journal of the Atmospheric Sciences, vol. 20, no. 2, pp. 130-1341, 1963.

[2] V. Afraimovic, V. Bykov, and L. P. Shilnikov, "Origin and structure of the Lorenz attractor," Soviet Physics-Doklady, vol. 22, pp. 253-255, 1977.

[3] C. Sparrow, The Lorenz equations: bifurcations, chaos, and strange attractors, vol. 41 of Applied Mathematical Sciences, Springer, New York, NY, USA, 1982.

[4] M. F. Doherty and J. M. Ottino, "Chaos in deterministic systems: strange attractors, turbulence, and applications in chemical engineering," Chemical Engineering Science, vol. 43, no. 2, pp. 139-183, 1988.

[5] K. T. Alligood, T. D. Sauer, and J. A. Yorke, Chaos, Textbooks in Mathematical Sciences, Springer, New York, NY, USA, 1997.
[6] J. C. Sprott, "Some simple chaotic flows," Physical Review E, vol. 50, no. 2, pp. R647-R650, 1994.

[7] G. Chen and T. Ueta, "Yet another chaotic attractor," International Journal of Bifurcation and Chaos in Applied Sciences and Engineering, vol. 9, no. 7, pp. 1465-1466, 1999.

[8] A. Vaněček and S. Čelikovský, "Bilinear systems and chaos," Kybernetika, vol. 30, no. 4, pp. 403-424, 1994.

[9] S. Vaněček and A. Čelikovský, Control Systems: From Linear Analysis to Synthesis of Chaos, Prentice-Hall, London, UK, 1996.

[10] J. Lü and G. Chen, "A new chaotic attractor coined," International Journal of Bifurcation and Chaos in Applied Sciences and Engineering, vol. 12, no. 3, pp. 659-661, 2002.

[11] S. Čelikovský and G. Chen, "On the generalized Lorenz canonical form," Chaos, Solitons and Fractals, vol. 26, no. 5, pp. 12711276, 2005.

[12] Q. Yang, G. Chen, and T. Zhou, "A unified Lorenz-type system and its canonical form," International Journal of Bifurcation and Chaos in Applied Sciences and Engineering, vol. 16, no. 10, pp. 2855-2871, 2006.

[13] Q. Yang, G. Chen, and K. Huang, "Chaotic attractors of the conjugate Lorenz-type system," International Journal of Bifurcation and Chaos in Applied Sciences and Engineering, vol. 17, no. 11, pp. 3929-3949, 2007.

[14] K. Huang and Q. Yang, "Stability and Hopf bifurcation analysis of a new system," Chaos, Solitons and Fractals, vol. 39, no. 2, pp. 567-578, 2009.

[15] Q. Zhang, J. H. L. Lü, and S. H. Chen, "Coexistence of antiphase and complete synchronization in the generalized Lorenz system," Communications in Nonlinear Science and Numerical Simulation, vol. 15, no. 10, pp. 3067-3072, 2010.

[16] X. Shi and Z. Wang, "The alternating between complete synchronization and hybrid synchronization of hyperchaotic Lorenz system with time delay," Nonlinear Dynamics, vol. 69, no. 3, pp. 1177-1190, 2012.

[17] S. Camargo, L. R. L. Viana, and C. Anteneodo, "Intermingled basins in coupled Lorenz systems," Physical Review E, vol. 85, no. 3, Article ID 036207, 10 pages, 2012.

[18] S. Li, Y. Li, B. Liu, and T. Murray, "Model-free control of Lorenz chaos using an approximate optimal control strategy," Communications in Nonlinear Science and Numerical Simulation, vol. 17, no. 12, pp. 4891-4900, 2012.

[19] S. Čelikovský and G. Chen, "On a generalized Lorenz canonical form of chaotic systems," International Journal of Bifurcation and Chaos in Applied Sciences and Engineering, vol. 12, no. 8, pp. 1789-1812, 2002.

[20] S. Čelikovský and G. Chen, "Hyperbolic-type generalized Lorenz system and its canonical form," in Proceedings of the 15th Triennial World Congress of IFAC, Barcelona, Spain, July 2002.

[21] I. Grigorenko and E. Grigorenko, "Chaotic dynamics of the fractional order Lorenz system," Physical Review Letters, vol. 91, no. 3, Article ID 03410, 4 pages, 2003.

[22] C. P. Li and G. J. Peng, "Chaos in Chen's system with a fractional order," Chaos, Solitons and Fractals, vol. 22, no. 2, pp. 443-450, 2004.

[23] J. P. Yan and C. P. Li, "On chaos synchronization of fractional differential equations," Chaos, Solitons and Fractals, vol. 32, no. 2, pp. 725-735, 2007.

[24] P. Zhou P and X. Cheng, "Synchronization between different fractional order chaotic systems," in Proceeding of the 7th World Congress on Intelligent Control and Automation, Chongqing, China, June 2008. 
[25] Y. Yu, H. X. Li, S. Wang, and J. Yu, "Dynamic analysis of a fractional-order Lorenz chaotic system," Chaos, Solitons and Fractals, vol. 42, no. 2, pp. 1181-1189, 2009.

[26] K. Sun and J. C. Sprott, "Bifurcations of fractional-order diffusionless lorenz system," Electronic Journal of Theoretical Physics, vol. 6, no. 22, pp. 123-134, 2009.

[27] S. K. Agrawal, M. Srivastava, and S. Das, "Synchronization of fractional order chaotic systems using active control method," Chaos, Solitons and Fractals, vol. 45, no. 6, pp. 737-752, 2012.

[28] Y. Xu, R. Gu, H. Zhang, and D. Li, "Chaos in diffusionless Lorenz system with a fractional order and its control," International Journal of Bifurcation and Chaos, vol. 22, no. 4, pp. 1-8, 2012.

[29] P. Zhou and R. Ding, "Control and synchronization of the fractional-order Lorenz chaotic system via fractionalorder derivative," Mathematical Problems in Engineering, vol. 2012, Article ID 214169, 14 pages, 2012.

[30] G. Si, Z. Sun, H. Zhang, and Y. Zhang, "Parameter estimation and topology identification of uncertain fractional order complex networks," Communications in Nonlinear Science and Numerical Simulation, vol. 17, no. 12, pp. 5158-5171, 2012.

[31] A. C. Fowler, M. J. McGuinness, and J. D. Gibbon, "The complex Lorenz equations," Physica D, vol. 4, no. 2, pp. 139-163, 1981/82.

[32] A. C. Fowler, J. D. Gibbon, and M. J. McGuinness, "The real and complex Lorenz equations and their relevance to physical systems," Physica D, vol. 7, no. 1-3, pp. 126-134, 1983.

[33] C. Z. Ning and H. Haken, "Detuned lasers and the complex Lorenz equations: subcritical and supercritical Hopf bifurcations," Physical Review A, vol. 41, no. 7, pp. 3826-3837, 1990.

[34] A. D. Kiselev, "Symmetry breaking and bifurcations in complex Lorenz model," Journal of Physical Studies, vol. 2, no. 1, pp. 3037, 1998.

[35] A. Rauh, L. Hannibal, and N. B. Abraham, "Global stability properties of the complex Lorenz model," Physica D, vol. 99, no. 1, pp. 45-58, 1996.

[36] S. Panchev and N. K. Vitanov, "On asymptotic properties of some complex Lorenz-like systems," Journal of the Calcutta Mathematical Society, vol. 1, no. 3-4, pp. 121-130, 2005.

[37] G. M. Mahmoud, M. A. Al-Kashif, and S. A. Aly, "Basic properties and chaotic synchronization of complex Lorenz system," International Journal of Modern Physics C, vol. 18, no. 2, pp. 253-265, 2007.

[38] G. M. Mahmoud, M. E. Ahmed, and E. E. Mahmoud, "Analysis of hyperchaotic complex Lorenz systems," International Journal of Modern Physics C, vol. 19, no. 10, pp. 1477-1494, 2008.

[39] E. E. Mahmoud and G. M. Mahmoud, Chaotic and Hyperchaotic Nonlinear Systems, Lambert Academic Publishing, Saarbrücken, Germany, 2011.

[40] G. M. Mahmoud and E. E. Mahmoud, "Complete synchronization of chaotic complex nonlinear systems with uncertain parameters," Nonlinear Dynamics, vol. 62, no. 4, pp. 875-882, 2010.

[41] Z. Li, Z. Duan, L. Xie, and X. Liu, "Distributed robust control of linear multi-agent systems with parameter uncertainties," International Journal of Control, vol. 85, no. 8, pp. 1039-1050, 2012.

[42] E. E. Mahmoud, "Dynamics and synchronization of new hyperchaotic complex Lorenz system," Mathematical and Computer Modelling, vol. 55, no. 7-8, pp. 1951-1962, 2012.

[43] A. M. A. El-Sayed, E. Ahmed, and H. A. A. El-Saka, "Dynamic properties of the fractional-order logistic equation of complex variables," Abstract and Applied Analysis, vol. 2012, Article ID 251715, 12 pages, 2012.

[44] H. Haken, "Analogy between higher instabilities in fluids and lasers," Physics Letters A, vol. 53, no. 1, pp. 77-88, 1975.

[45] X. Li and W. Chen, "Analytical study on the fractional anomalous diffusion in a half-plane," Journal of Physics A, vol. 43, no. 49, Article ID 495206, 11 pages, 2010.

[46] Y. J. Liang and W. Chen, "A survey on numerical evaluation of Lvy stable distributions and a new MATLAB toolbox," Signal Processing, vol. 93, no. 1, pp. 242-251, 2013.

[47] S. Hu, W. Chen, and X. Gou, "Modal analysis of fractional derivative damping model of frequency-dependent viscoelastic soft matter," Advances in Vibration Engineering, vol. 10, no. 3, pp. 187-196, 2011.

[48] I. Podlubny, Fractional Differential Equations, vol. 198 of Mathematics in Science and Engineering, Academic Press, London, UK, 1999.

[49] A. A. Kilbas, H. M. Srivastava, and J. J. Trujillo, Theory and Applications of Fractional Differential Equations, vol. 204 of North-Holland Mathematics Studies, Elsevier Science B.V., Amsterdam, The Netherlands, 2006.

[50] J. Sabatier, O. P. Agrawal, and J. A. Machado, Advance in Fractional Calculus: Theoretical Developments and Applications in Physics and Engineering, Springer, Dordrecht, The Netherlands, 2007.

[51] L. Chen, Y. Chai, R. Wu, and J. Yang, "Stability and stabilization of a class of nonlinear fractional order system with Caputo derivative," IEEE Transaction on Circuits and Systems, vol. 59, no. 9, pp. 602-606, 2012.

[52] K. Diethelm and N. J. Ford, "Analysis of fractional differential equations," Journal of Mathematical Analysis and Applications, vol. 265, no. 2, pp. 229-248, 2002.

[53] D. Matignon, "Stability result on fractional differential equations with applications to control processing," in Proceedings of the IMACS-SMC 96, vol. 2, pp. 963-968, 1996. 


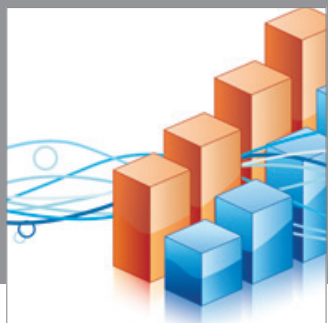

Advances in

Operations Research

mansans

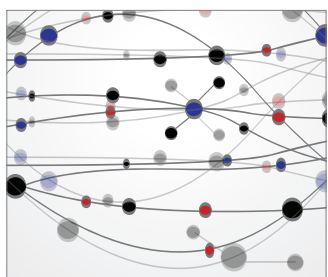

The Scientific World Journal
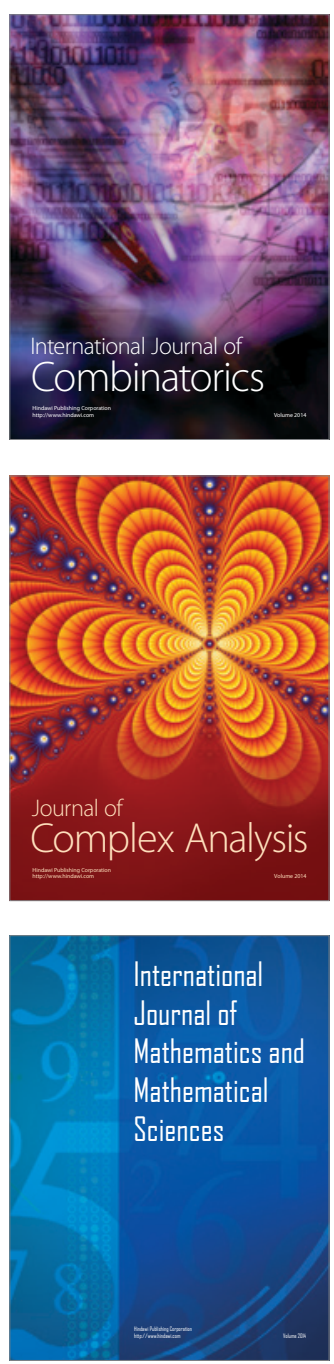
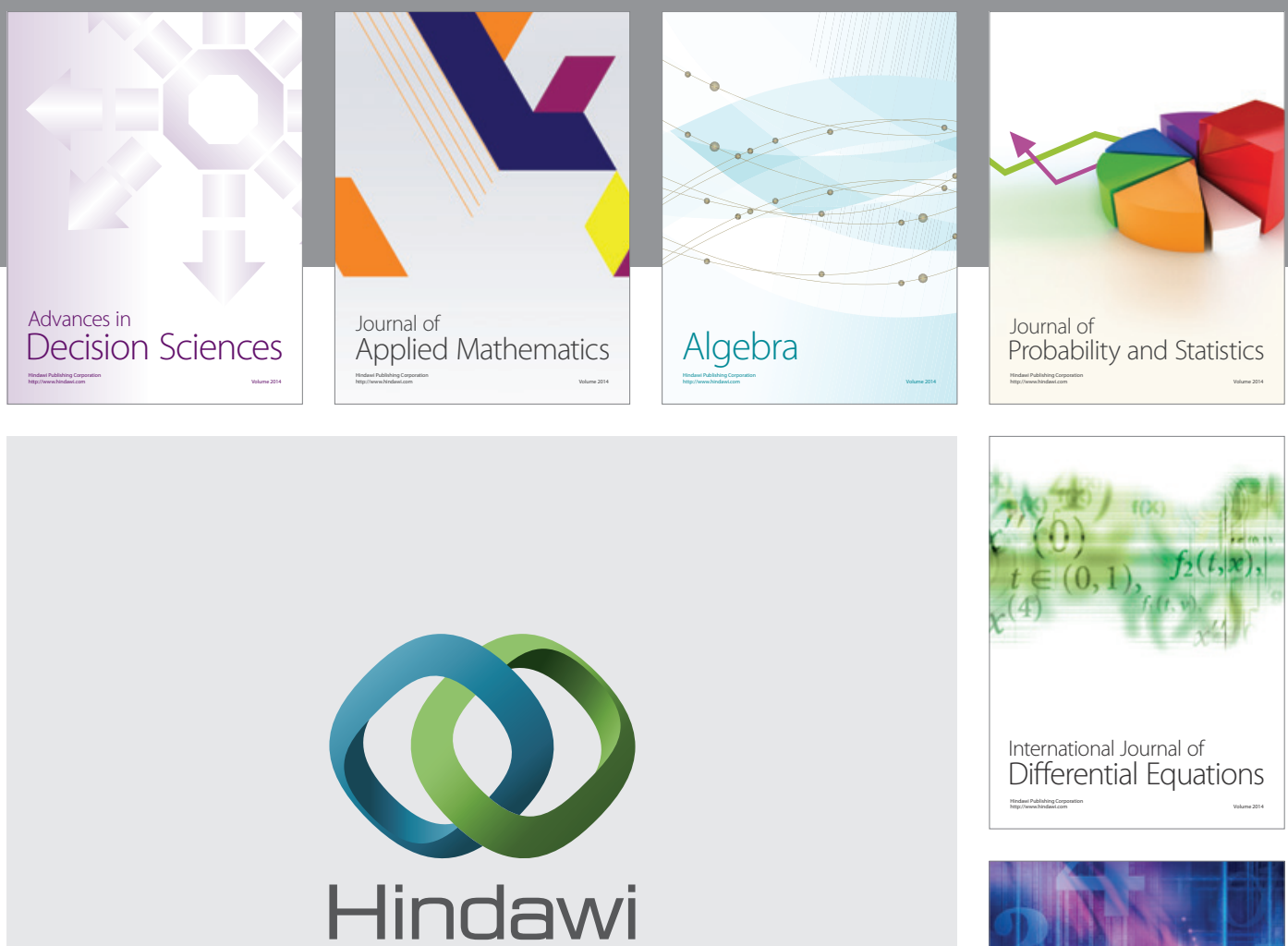

Submit your manuscripts at http://www.hindawi.com
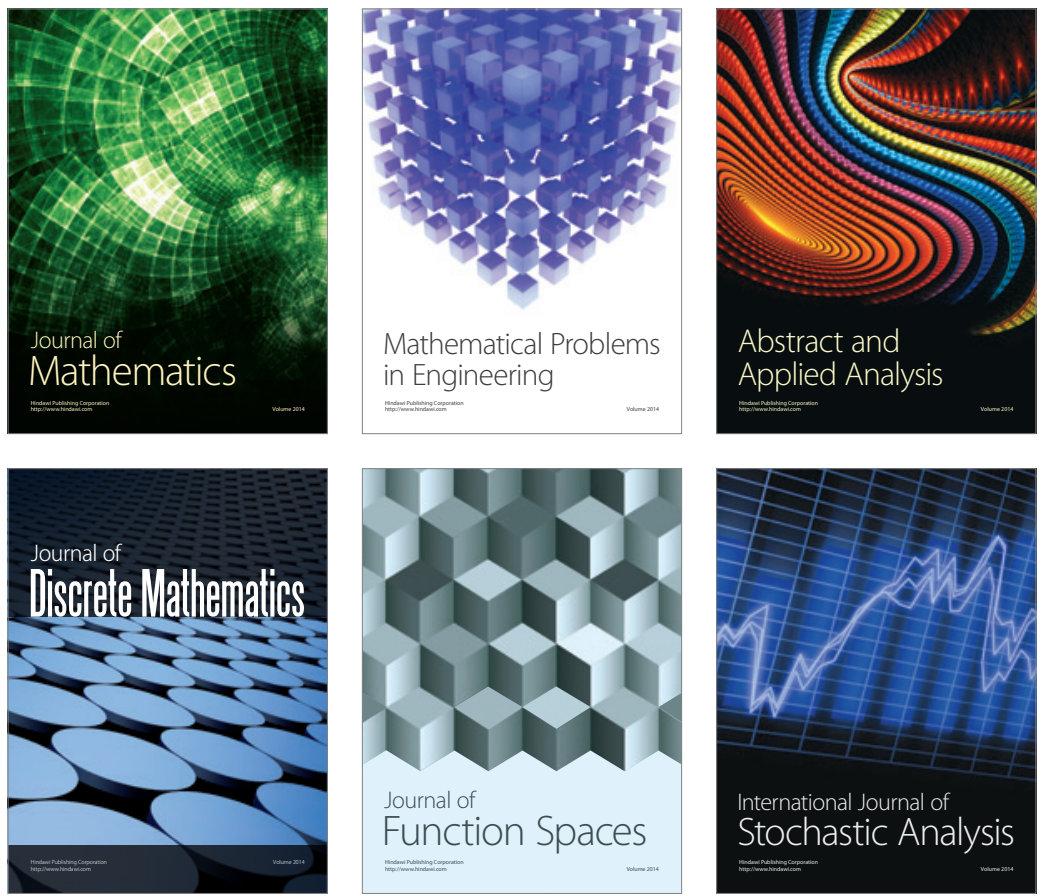

Journal of

Function Spaces

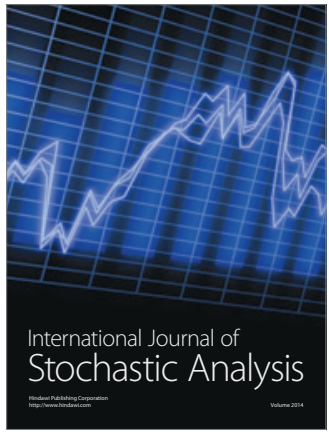

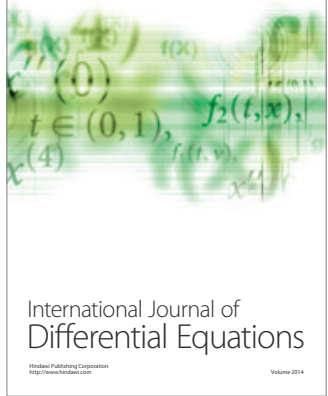
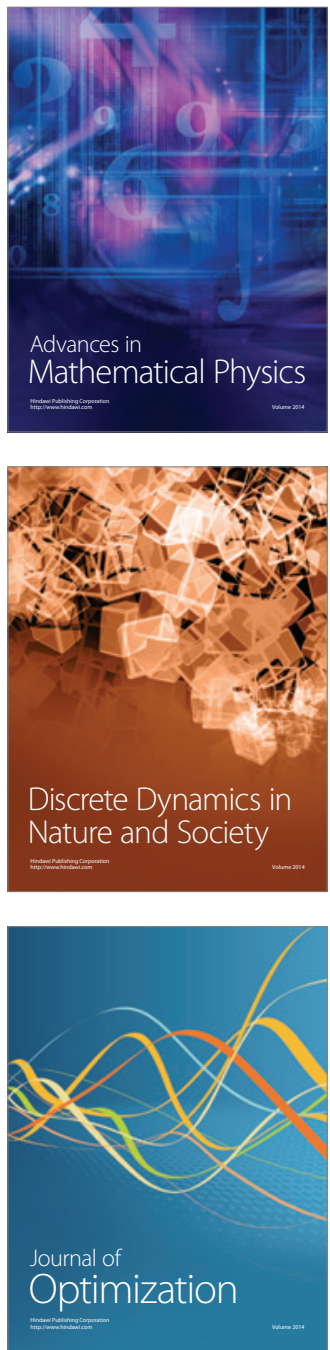